\title{
Impact of Raman scattering and modulation instability on the performances of Brillouin sensors
}

\author{
Stella M. Foaleng ${ }^{1}$ and Luc Thévenaz \\ EPFL Swiss Federal Institute of Technology, Group for Fibre Optics, Institute of Electrical Engineering, \\ STI IEL GR-SCI Station11, CH-1015 Lausanne, Switzerland
}

\begin{abstract}
The impact of Raman scattering and modulation instability is studied in Brillouin time-domain analysis systems. It turns out to be very detrimental for long-range sensing as a result of the extended interaction length combined to the high pump peak pulse power. The conditions under which these effects limit the sensing range are determined and the modeling is very well confirmed by experimental results.
\end{abstract}

Keywords: Fibre optics, optical fibre sensor, stimulated Brillouin scattering, distributed fibre sensor, non-linear optics, stimulated Raman scattering, modulation instability.

\section{INTRODUCTION}

The recent raising interest in Brillouin fiber sensors for application in civil engineering, in oil \& gas industry, in perimeter security and in intrusion detection has stimulated the research efforts to extend the detection range. In timedomain distributed Brillouin sensors (BOTDA) pulses are used to interrogate locally the interaction along the fiber. In order to achieve better performances in terms of range and spatial resolution, pump pulse and signal powers must be raised. These powers cannot be made arbitrarily large and must be kept below the observation threshold of any other noise-fed nonlinear effects. In these systems the most critical nonlinear effects are those in which a signal wave is amplified through a forward interaction, in other words when the background noise is amplified while co-propagating with the pump pulse. In such a situation the interaction length may cover many tens of kilometers and even a weak nonlinear amplification much less efficient than stimulated Brillouin scattering (SBS) may result in a large amplified spontaneous signal that eventually depletes the pump. The identified nonlinear effects are the modulation instability (MI), which occurs only in fibers presenting an anomalous dispersion at the pump wavelength, and the Raman scattering (RS). A spectral self-broadening of the pump due to MI was observed in sensors based on spontaneous Brillouin scattering ${ }^{[1]}$ whereas a rapid pump depletion was observed in the case of BOTDA sensing system ${ }^{[2]}$. Similar observations were realized in the case of RS in which the Brillouin gain was observed to abruptly fall to zero ${ }^{[3]}$ after several kilometers, severely limiting the distance range that turns highly dependent on the pump pulse power. Whereas the former works ${ }^{[3]-[2]}$ have shown a correct intuition based on experimental observations to address qualitatively the issue, we present here a quantitative model to anticipate the detrimental impact of MI and RS, validated by a clear experimental demonstration.

\section{THEORY}

Raman scattering ${ }^{[4]}$ has fundamental similarities with Brillouin scattering, both resulting from the interaction between light and phonons, though showing distinct features. The most noticeable difference is the frequency shift experienced by the scattered light (13 THz for Raman, $11 \mathrm{GHz}$ for Brillouin scattering), but another important distinct feature is the less strict phase matching condition in RS that makes possible forward scattering. In this latter case the input pump pulse and the Raman-generated signal are co-propagative and may interact over long distance or even the entire fiber length (unlike the case of the backward-only Brillouin scattering where the interaction length is limited to the pump pulse width). A very similar behavior is observed for modulation instability, which is a consequence of the interaction between Kerr effect and anomalous dispersion, giving rise to a train of solitons self-building from noise. In that case the signal manifests as spectral side lobes around the pump frequency that extends over many tens of GHz, depending on the pump power and the medium chromatic dispersion that must be anomalous $\left(D_{\lambda}>0\right)$.

The modeling of a signal building from noise through forward Raman scattering has been proposed by Smith ${ }^{[5]}$ in 1972 and has proved to be robust. In our modeling we use the expressions derived by Smith for forward Raman scattering and

${ }^{1}$ Email: stella.foalengmafang@epfl.ch; phone+41 21693 7387; fax +41 216934660

21st International Conference on Optical Fiber Sensors, edited by Wojtek J. Bock, Jacques Albert, Xiaoyi Bao, Proc. of SPIE Vol. 7753, 77539V · C 2011 SPIE · CCC code: 0277-786X/11/\$18 · doi: 10.1117/12.885105 
interpret them in the particular context of distributed Brillouin sensors. Then we adapt this modeling to the particular case of modulation instability, following the same approach, to establish new relations valid to predict the observation threshold for MI.

Let us consider a fiber with a nonlinear effective area $A_{\text {eff }}$ and of length $L$ with a linear attenuation $\alpha$. Let the pump pulse enter the fiber at $z=0$, traveling in the $+z$ direction with a power $P_{p}$ and an effective intensity $I_{p}=P_{p} / A_{\text {eff }}$. Under forward Raman amplification, the output signal power $P_{s}$ due to amplified spontaneous Raman scattering produced by the incident pump intensity for an effective nonlinear distance $L_{\text {eff }}=(1-\exp (\alpha L)) / \alpha$ is scaled by the peak Raman gain coefficient $g_{R}$ and its effective bandwidth:

$$
B_{e f f}=\frac{\sqrt{\pi}}{2} \frac{\Delta v_{\mathrm{FWHM}}}{\sqrt{g_{R} I_{p}(0) L_{e f f}}},
$$

where $\Delta v_{\mathrm{FWHM}}$ is the full width at half maximum of the spontaneous gain spectrum. A critical pump power $P_{\text {crit }}$ is defined as the fictitious case where this pump amplifies the spontaneous signal to make it as large as the pump power, deliberately neglecting the effect of pump depletion. Actually this critical power $P_{\text {crit }}$ is a good estimation of the pumping level giving rise to an amplified noise with power comparable to the pump and thus evidently depleting the pump. This leads to the following nonlinear equation to be solved for $P_{\text {crit }}$ :

$$
\frac{\sqrt{\pi}}{2}\left(h v_{s}\right) \frac{g_{R}}{A_{e f f}} L_{e f f} \Delta v_{\mathrm{FWHM}}=\left[\frac{g_{R} P_{\text {crit }} L_{e f f}}{A_{\text {eff }}}\right]^{3 / 2} \exp \left[-\frac{g_{R} P_{\text {crit }} L_{e f f}}{A_{\text {eff }}}\right],
$$

from which an approximate simplified equality can be obtained using the Raman spectral characteristics in silica fibers:

$$
P_{\text {crit }}^{R} \approx \frac{16 A_{\text {eff }}}{g_{R} L_{\text {eff }}},
$$

In total analogy a similar relation can be established for MI. The essential step is to determine the effective bandwidth $B_{\text {eff }}$ that is directly related to the spectral characteristics of the broadband gain due to MI. From the analysis of the nonlinear Schrödinger equation for anomalous dispersion, we can find the MI gain spectrum $G(\omega)=\left|\beta_{2} \omega\right| \sqrt{\omega_{c}^{2}-\omega^{2}}$, where $\omega_{c}=\left(4 \gamma P_{p} / \beta_{2}\right)^{1 / 2}$ is the critical frequency, $\beta_{2}$ the dispersion coefficient, $\gamma$ the nonlinear parameter and $P_{p}$ the input power. The maximum gain is $G_{\max }=2 \gamma P_{p}$. From this gain spectrum the effective bandwidth $B_{\text {eff }}$ can then be calculated:

$$
B_{e f f}=\frac{1}{\pi} \sqrt{\frac{4 \gamma P_{p}}{\left|\beta_{2}\right|}},
$$

to establish a nonlinear equation for the critical power similar to Eq.2

$$
\frac{1}{\pi}\left(h v_{s}\right) \sqrt{\frac{2}{\beta_{2} L_{e f f}}}=\sqrt{2 \gamma P_{\text {crit }} L_{e f f}} \exp \left[-2 \gamma P_{\text {crit }} L_{e f f}\right]
$$

that also leads to a simplified equality valid to determine the critical power for modulation instability under the condition of anomalous dispersion $\left(\beta_{2}<0\right)$ :

$$
P_{c r i t}^{M I} \approx \frac{20}{2 \gamma L_{e f f}},
$$

In standard fibers at $1550 \mathrm{~nm}$ the dispersion is anomalous and $P_{\text {crit }}^{M I} \square 0.78 P_{\text {crit }}^{R}$ using standard parameters, so that MI will always be observable at lower power than RS and will be therefore the dominating limitation.

\section{EXPERIMENTAL RESULTS}

These theoretical expressions were compared to experimental data obtained using a classical Brillouin optical timedomain analyzer based on a high performance pump and probe configuration ${ }^{[6]}$. The duration of the rectangular pump pulses (30 ns corresponding to $3 \mathrm{~m}$ spatial resolution) was carefully chosen to produce clean signals with an important gain for a better demonstration. The probe power is kept low enough at $56 \mu \mathrm{W}$ to avoid any depletion effect resulting from the Brillouin interaction.

We investigated the effect of MI using a $25.5 \mathrm{~km}$ standard SM fiber with relevant characteristics listed in Table 1 . The fiber length is longer than the maximum nonlinear length $1 / \alpha=22 \mathrm{~km}$, so that results can be considered as representative for any very long fibers. To observe the MI sidebands appearing symmetrically around the pump frequency, we 


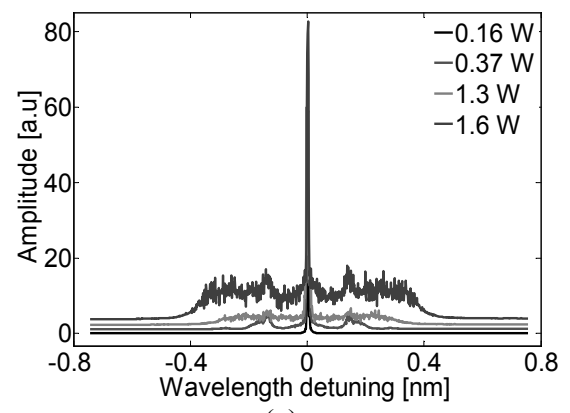

(a)

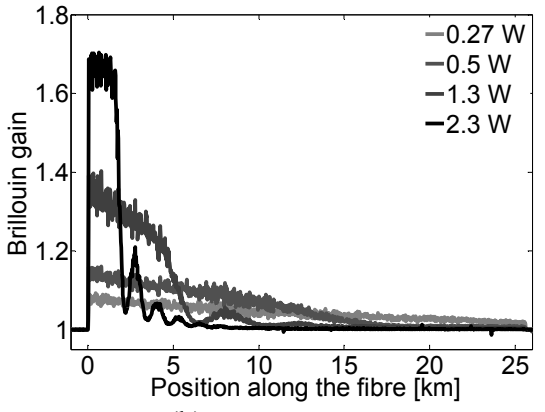

(b)

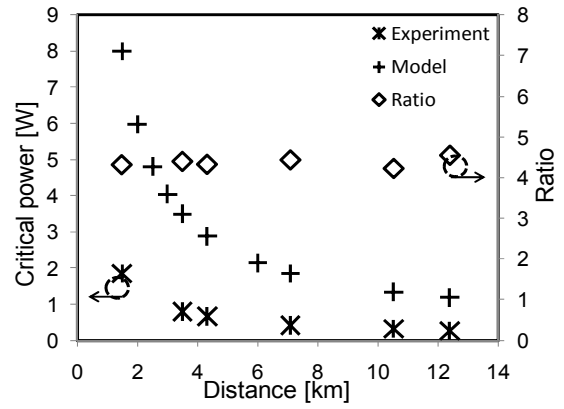

(c)

Fig. 1: (a) Measured power spectra of MI at the far end of a $25.5 \mathrm{~km}$ SMF in the anomalous dispersion regime. (b) Distributed Brillouin gain measurements in a $25.5 \mathrm{~km}$ SMF fibre showing the pump depletion due to MI after a critical distance depending on the pump peak power; (c) Experimental $(*)$ and theoretical $(+)$ critical power as a function of the critical distance where the pump depletion turns significant (Brillouin gain fall-off) and the ratio $(\diamond)$ between theoretical and experimental values.

measured series of power spectra using an optical spectrum analyzer at the output fiber end for various input pulse peak power $(\sim 160 \mathrm{~mW}$ to $1.6 \mathrm{~W})$, shown in Fig. 1a. It can be seen that the MI effect becomes more pronounced as the power increases. The sidebands which appear symmetrically around the pump frequency become broader for higher pump power, leading gradually to pump depletion. The influence of MI on the distributed Brillouin amplification was then measured by varying the input pump peak power from $270 \mathrm{~mW}$ to $2.3 \mathrm{~W}$. Fig. 1b shows the effective Brillouin gain as a function of the position in a $25.5 \mathrm{~km}$ SM fiber for four pump peak powers. The graph demonstrates clearly the gradual drop of the gain after $10.52 \mathrm{~km}$ for a $500 \mathrm{~mW}$ pump that turns more pronounced and at shorter distance for higher pump powers. It can be seen that no gain fall-off is observed at $270 \mathrm{~mW}$ pump power, so we can deduce the absence of MI at this power level. Since MI can be interpreted in the spectral domain as resulting from an energy transfer between the fundamental mode constituting the initial continuous wave and high-order modes ${ }^{12}$, several theoretical studies have shown that, after a definite propagation length, the energy spread over different frequencies eventually returns to the initial mode ${ }^{[7]-[8]}$ (this reversible behavior of MI is known as Fermi-Pasta-Ulam recurrence FPU). This is experimentally observed in Fig. 1b for pump power much higher than $500 \mathrm{~mW}$ in which a ripple is observed. In Fig. 1c is represented the pump power as a function of a critical distance evaluated as the position where the Brillouin gain starts to drop. On the same graph is calculated from the model the critical power $P_{c r i t}^{M I}$ using Eq.6 for each critical distance measured experimentally. Finally the ratio between these theoretical and experimental values is represented and takes a constant value close to 4 . This shows that the critical power given by the model is over-evaluated and in the reality the depletion effect on the pump by MI takes place at a power lower than the value given by the model critical power. The discrepancy may come from the fact that the MI emission keeps spreading over a broadband, as illustrated in Fig. 1a, and no clear dynamic spectral narrowing of the amplified spontaneous emission is observed, so that a corrective empirical factor must be preferably inserted in Eq.6 to give a good prediction, yielding this final expression:

$$
P_{c r i t}^{M I} \approx \frac{5}{2 \gamma L_{e f f}},
$$

Nevertheless the fact that the ratio keeps constant for a large range of pump powers proves the robustness of the model. So finally, $P_{c r i t}^{M I} \square 0.2 P_{c r i t}^{R}$ and MI will always be observable at lower power than RS and remains the dominating limitation.

To evaluate the impact of forward Raman scattering, it is necessary to avoid modulation instability by propagating the pump in normal dispersion conditions. For this purpose a $13 \mathrm{~km}$ DSF fiber was used and the same set of measurements at different powers was carried out. It was checked that the present depletion was purely due to RS by observing the presence of a strong signal at $1672 \mathrm{~nm}$ and by observing the depletion of the pump pulse at the initial wavelength, as shown in Fig. 2a. The pump pulse undergoes strong distortions as the input pump power increases and the group velocity difference at the pump and Raman wavelength causes a differential depletion over the pulse duration, even leaving a very short un-depleted section at the front edge of the pulse. Fig. $2 b$ shows the effective Brillouin gain as a function of the position in the DSF fiber for a pump peak power from $\sim 400 \mathrm{~mW}$ to $2.16 \mathrm{~W}$. In total similarity with MI

Table 1: Characteristics of the SMF and the DSF fibers used for the experiments.

\begin{tabular}{|l|l|l|l|l|}
\hline Fibre type & Fibre loss $(\mathrm{dB} / \mathrm{km})$ & Effective area $\left(\mu \mathrm{m}^{2}\right)$ & Dispersion $@ 1550 \mathrm{~nm}(\mathrm{ps} / \mathrm{km} \mathrm{nm})$ & Raman Gain coef. $(1 / \mathrm{mW})$ \\
\hline SMF & 0.22 & $\sim 80$ & 17 & $\sim 1 \mathrm{e}^{-13}$ \\
\hline DSF & 0.14 & $\sim 50$ & -2 & $\sim 4 \mathrm{e}^{-13}$ \\
\hline
\end{tabular}




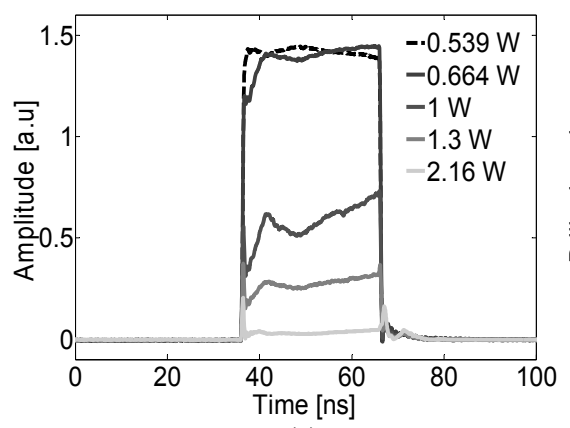

(a)

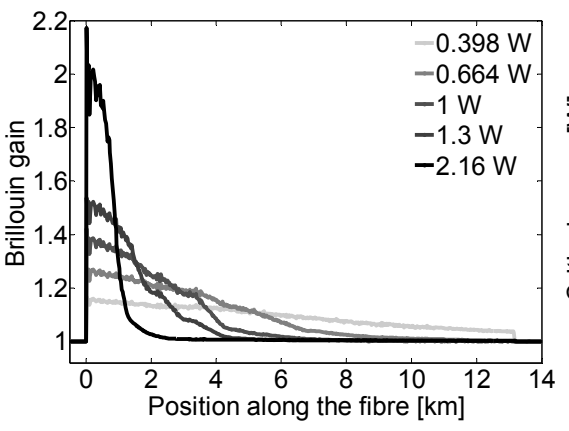

(b)

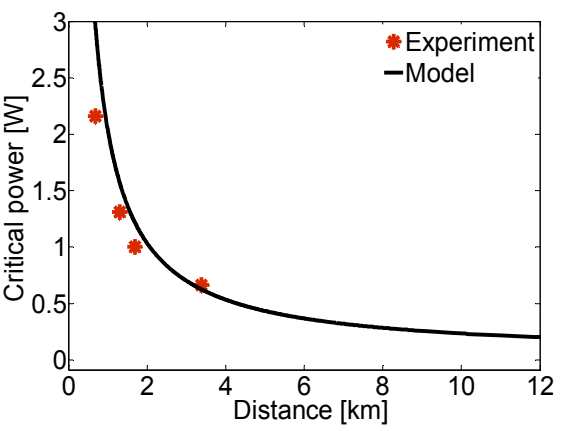

(c)

Fig. 2: (a) The corresponding time domain measurement waveform of the pump pulse at the output of a $13 \mathrm{~km}$ DSF for different pump peak powers; (b) Brillouin gain distributed measurement showing the pump depletion by Raman scattering in a $13 \mathrm{~km}$ DSF for different input pump peak powers; (c) Maximum distance before Raman scattering begins to deplete the pump: experimental points $(*)$, and theoretical model (solid line).

the graph shows clearly the gradual drop of the gain after $3.4 \mathrm{~km}$ for a $664 \mathrm{~mW}$ pump power which turns more pronounced and abrupt for higher pump powers. The absence of Raman effect is observed below a $400 \mathrm{~mW}$ pump power, so at pump powers higher than MI as anticipated by the model. Fig. 2c represents the graph of the pump power versus critical distance for experimental data $(*)$ and for model prediction using Eq.3 (solid line) showing an excellent agreement between the experimental result and the theoretical expression (using the fiber characteristics shown in Table 1).

\section{CONCLUSION}

In this paper we have investigated the undesirable effects of modulation instability and forward Raman scattering in distributed BOTDA sensors systems. Both effects are detrimental and have been evaluated experimentally and compared to a theoretical model giving a simplified expression to predict the critical power for a given distance range. In a practical sensor the critical distance must be longer than the actual fiber length and is bound by the nonlinear fiber length, fixing a maximum pump power. MI turns out to be the dominant nonlinear limitation since it shows the lowest critical power, but it can be avoided to a wide extent by using the fiber in the normal dispersion spectral region such as a DSF in the C-band. On the other hand Raman scattering can be avoided only by limiting the optical pump power and therefore is the ultimate nonlinear limitation in a distributed sensing system. Under similar conditions RS shows a critical power $\sim 5$ times larger than MI.

\section{ACKNOWLEDGEMENTS}

The authors address their warm thanks to Prof. Jie Lin from Bucknell University for her critical comments about the model for Raman scattering. This study was realized in the framework of the European COST Action 299 "FIDES", supported by the project SER COST C06.0015 from the Swiss Science National Foundation.

\section{REFERENCES}

[1] Alahbabi M.N., Cho Y. T. and Newson T. P. "Influence of modulation instability on distributed optical fibre sensors based on spontaneous Brillouin scattering, " J. Opt. Soc. Am. B (21)6, 1156-1160 (2004).

[2] D. Alasia et al. "Detrimental effect of modulation instability on distributed optical fibre sensors using stimulated Brillouin scattering" Proc. SPIE 5855, 587-590 (2005).

[3] Fellay A., Thévenaz L., Facchini M. and Robert P. "Limitation of Brillouin time-domain analysis by Raman scattering," Proc. of the 5th Opt. Fibre Meas. Conf., Nantes, France, 110-113 (1999).

[4] G. Agrawal, Nonlinear Optics, fourth ed. (Academic, 2008).

[5] Smith R.G., "Optical Power Handling Capacity of Low Loss Optical Fibers as Determined by Stimulated Raman and Brillouin Scattering," App. Opt. 11(11), 2489-2494 (1972).

[6] S. Diaz, S. M. Foaleng, M. Lopez-Amo, and L. Thévenaz, "A High-Performance Optical Time-Domain Brillouin Distributed Fiber sensor", IEEE Sensors journal, 8, 1268-1272 (2008).

[7] E. Fermi, J. Pasta, H. C. Ulam, "Studies of nonlinear problems", in Collected Papers of Enrico Fermi, E. Serge Ed., 2, 977-988 (1965).

[8] G.V. Symaeys, Ph. Emplit, M. Haelterman, "Experimental demonstration of the Fermi-Pasta-Ulam recurrence in a modulationally unstable optical wave", Phys. Rev. Lett., 87, 3 (2001). 